\title{
Modelling the motion of clusters of cells in a viscous fluid using the boundary integral method.
}

\author{
Paul J. Harris
}

School of Computing, Engineering and Mathematics, University of Brighton, Lewes Road, Brighton, UK.

\begin{abstract}
In experiments clusters of cells are often observed to move in response to a chemical signal which is present in the fluid surrounding the cells. This process is known as chemotaxis. This paper presents a method for modelling the motion of clusters of cells moving through a viscous fluid in response to a known chemical signal using a boundary integral formulation of the governing equations rather than the more usual differential equation formulation. The numerical results presented in this paper show that the boundary integral method can be used to simulate the motion of cell clusters through the fluid. The results of the simulations are compared to some experimental observations of cell and cluster motion.

Keywords: Chemotaxis, Mathematical Modelling, Boundary Integral Method, Stokes Flow
\end{abstract}

\section{Introduction}

This paper is concerned with simulating how clusters of cells move through a fluid medium in response to changes in the concentration of a chemical that is present in the fluid. In some cases the chemical is a protein secreted by the cells themselves to attract other cells (or clusters) in order to combine and form larger clusters. However, in other situations the chemical is simply present in the environment containing the cells and they just react to its presence. Over the years, a number of papers have been published which illustrate experiments where chemotaxis has been observed (see [1,2, $3,4]$ for example). Further, the aggregation of cells into clusters has been reported in the literature. For example, Jia et. al [5] report on the aggregation of rodent pancreatic cells, and Hilderink et. al. [6] studied the aggregation of human pancreatic cells into clusters.

The mathematical modelling of chemotaxis has been an active topic of research in recent years. The models that have been developed can be broadly classified as either those where density of the cells is modeled or those where the motion of each individual cell or cluster is modeled. Models which consider the concentration or density of the cells use the diffusion or diffusion-reaction equation to model how both the density of the cells in their environment and the concentrations of the chemical signal evolve through time, see $[7,8,9,10]$ for example. These models are often referred to as KellerSegel models in the literature. However, these models do not give any information on 
how each cell or cluster of cells move. They also have the limitation that they do not include the effects of the motion of the surrounding fluid, although the model proposed by Deleuze et. al. [11] does include some effects of the fluid motion.

In other models, the motion of each individual cell or cluster of cells is considered. In some of these models the cells are represented by simple geometric shapes such in the models proposed by $[12,13,14]$ for example. Harris [15] has developed a simple model of how small clusters of cells combine to form larger clusters. The simple model presented in [15] has only a superficial model of the fluid damping and does not include the fluid motion due to the motion of the cells, but does have the advantage of being able to simulate the motion of a large number of cells and clusters with relatively little computational cost.

In all of the models discussed above the cells are assumed to be rigid in the sense that they do not change shape as they move through the fluid. There is some experimental evidence that this is the case (see the images in Nitta et. al [3] for example). Other more sophisticated models of how a cell detects the gradient of the chemical at its outer membrane have been developed, such with the finite element type model described in Elliott et. al. [16]. However, many of these models do not include the motion of the fluid surrounding the cells.

The mathematical model presented here considers the motion of cells and clusters of cells in an incompressible viscous fluid. For simplicity, the cells and clusters are assumed to be rigid bodies in the sense that they do not change shape as they move. Further, it is assumed that the fluid domain is a thin layer so that the vertical motion of the fluid and the vertical variation of the concentration of the chemical can be neglected meaning that the problem only needs to be solved in two space dimensions. Experimentally, this corresponds to modelling the motion of cells and clusters of cells as seen through a typical microscope. Finally, the fluid is assumed to be incompressible and at very low Reynolds number so that at any given instant in time the fluid motion can be represented by a Stokes flow.

Mathematically, there are advantages to assuming that clusters of cells have simple geometrical shapes. It is often possible to obtain a relatively simple analytical solution to the governing differential equations for quantities such as the exterior fluid velocity for such shapes. However, in most cases the clusters of cells do not form simple geometrical shapes (see the figures in Laganenka et. al [4] for example) and numerical methods have to be used for finding the approximate values of quantities such as the fluid velocity. A number of domain based methods, such as the finite element method or finite difference method, could be used to obtain the approximate solution of the governing differential equations. However, such methods require that the whole domain of the differential equation is gridded or meshed for the calculations, and that at each time-step the domain will need to be remeshed to account for the changes to the positions of the clusters.

To avoid these problems with the domain based methods an alternative approach based on a boundary integral formulation of the problem is presented in this paper. The governing domain based differential equations are recast as a boundary integral equation defined on the boundaries of cells and clusters, and which can be solved numerically using the boundary element method. This formulation has the advantage that is leads to a considerable reduction in the size of the computational problem when 
compared to using a domain based method such as the finite element method. A further advantage is that whilst the boundary element equations will need to be recalculated at each time-step, the appropriate part of the boundary element mesh simply moves with each cluster meaning that there is no need to completely remesh the problem at every time-step.

\section{Mathematical Model}

This section introduces the mathematical model of the motion of the cells and clusters of cells in response to a changing chemical signal in the medium in which the cells are immersed. For simplicity and brevity this section will only describe the model for clusters of cells as a single cell can be considered as a cluster with just one cell in it.

Let $\Omega$ denote the fluid-filled region exterior to one or more clusters of cells immersed in the fluid, and let $\Gamma^{[i]}$ and $\mathbf{x}_{i}$ denote the boundary and the location of the centre of mass of the $i^{\text {th }}$ cluster respectively. Further, let $\mathbf{v}_{i}$ and $\omega_{i}$ denote the translational and angular velocities of the $i^{\text {th }}$ cluster where the rotation is assumed to be around the cluster's centre of mass.

Assuming that the fluid is incompressible, the equations for the velocity $\mathbf{u}$ of the fluid can be expressed as the continuity equation [17]

$$
\nabla \cdot \mathbf{u}=0
$$

for the conservation of mass, and the Navier-Stokes equation

$$
\rho\left(\frac{\partial \mathbf{u}}{\partial t}+\mathbf{u} \cdot \nabla \mathbf{u}\right)=-\nabla p+\mu \nabla^{2} \mathbf{u}+\rho \mathbf{b}
$$

which can be considered as an expression of Newton's second law for a small particle of the fluid. Here $\rho$ and $\mu$ are used to denote the density and dynamic viscosity of the fluid, $p$ is the pressure and $\mathbf{b}$ is a known body force (which is assumed to be zero in this work, but can be used to include effects such as gravity). Since the length scale of a typical cluster of cells is very small (typically of the order of $10^{-5} \mathrm{~m}$ ) and the time scales over which the cells move very long (typically large fractions of a hour), the Reynolds number for the flow is very small. This mean that the inertial terms on the left-hand side of (2) can be neglected and in the absence of any body forces (2) can be simplified to

$$
-\nabla p+\mu \nabla^{2} \mathbf{u}=\mathbf{0}
$$

At this point it is worth noting that (1) and (3) are usually referred to as the governing equations for a steady Stokes flow. However, in the application considered here the flow is not steady as the cell is moving and the fluid velocity field will move with the cell. However, for the reasons given above, the inertia terms in the Navier-Stokes equation can be neglected meaning that (1) and (3) can be used to determine the velocity field at any given instant but they have to be solved at every time that the velocity field is required. 
The boundary condition are given in the form of a no-slip condition on the boundary of the each cluster, so

$$
\mathbf{u}(\mathbf{x})=\mathbf{v}_{i}+\left[\begin{array}{c}
y-y_{i} \\
x-x_{i}
\end{array}\right] \omega_{i} \quad \mathbf{x} \in \Gamma^{[i]} .
$$

An additional outer boundary $\Gamma^{[0]}$ is needed to avoid the problems associated with the Stokes paradox, which states that there is no solution to (1) and (3) which satisfies the boundary conditions (4) and the condition that $\mathbf{u} \rightarrow \mathbf{0}$ as $|\mathbf{x}| \rightarrow \infty$ [17]. This outer boundary is chosen to be a long way from the cell clusters (which in an experimental situation would correspond to a petri-dish containing the cells) and the boundary condition $\mathbf{u}=\mathbf{0}$ is imposed on this outer boundary.

In two space dimensions it can shown that if the fluid velocity $\mathbf{u}$ satisfies (1) and (3) in a closed domain $\Omega$ with a piecewise smooth boundary curve $\Gamma$, then the velocity also satisfies the boundary integral equation [18]

$$
\oint_{\Gamma} T\left(\mathbf{x}, \mathbf{x}_{0}\right) \mathbf{u}(\mathbf{x}) d \Gamma(\mathbf{x})-\oint_{\Gamma} G\left(\mathbf{x}, \mathbf{x}_{0}\right) \mathbf{f}(\mathbf{x}) d \Gamma(\mathbf{x})=\frac{1}{2} \mathbf{u}\left(\mathbf{x}_{0}\right)
$$

where $\mathbf{f}$ denotes the surface forces,

$$
\left.\begin{array}{c}
T_{i j}\left(\mathbf{x}, \mathbf{x}_{0}\right)=-\frac{\mathbf{r} \cdot \mathbf{n}}{\pi r^{4}} r_{i} r_{j} \\
G_{i j}\left(\mathbf{x}, \mathbf{x}_{0}\right)=\frac{1}{4 \pi \mu}\left(-\delta_{i j} \ln (r)+\frac{r_{i} r_{j}}{r^{2}}\right)
\end{array}\right\} i, j=1 \text { or } 2
$$

$\mathbf{r}=\mathbf{x}-\mathbf{x}_{0}, r=|\mathbf{r}|, \mathbf{n}$ is the unit normal to $\Gamma$ directed onto the the fluid domain $\Omega$ and $\delta_{i j}$ is the Kronecker delta function. Here $\Gamma$ is used to denote the union of the boundaries of the clusters and the outer fluid boundary. It should be noted that (5) is only valid for points $\mathbf{x}_{0}$ which are on a smooth part of $\Gamma$ and not at vertex of the boundary curve. For the piecewise constant boundary element method described below this is not a problem as $\mathbf{x}_{0}$ will alway be chosen to be on a smooth part of the curve. However, if a higherorder approximation to the solution, such a piecewise linear or piecewise quadratic, were used then this would need to be considered as the collocation points for such methods can potentially be located at a vertex of the boundary curve.

From the fluid velocity boundary conditions, the value of $\mathbf{u}$ is known on the whole of $\Gamma$, and hence (5) is a first kind Fredholm integral equation for the unknown force $\mathbf{f}$ on the boundary.

Here a piecewise constant collocation method has been used to solve (5) where the unknown force vector $\mathbf{f}$ is assumed to be constant within each boundary element. The boundary $\Gamma$ is divided into $N$ boundary elements $\Gamma_{1}, \Gamma_{2}, \cdots, \Gamma_{N}$ and the collocation point within each element is chosen to be the mid-point of the element. If $\mathbf{x}_{i}$ denotes the collation point for element $\Gamma_{i}$ then the boundary element approximation to (5) is

$$
\sum_{j=1}^{N} \mathbf{f}_{j} \int_{\Gamma_{j}} G\left(\mathbf{x}, \mathbf{x}_{i}\right) d \Gamma(\mathbf{x})=-\frac{1}{2} \mathbf{u}\left(\mathbf{x}_{i}\right)+\sum_{j=1}^{N} \int_{\Gamma_{j}} T\left(\mathbf{x}, \mathbf{x}_{i}\right) \mathbf{u}(\mathbf{x}) d \Gamma(\mathbf{x})
$$


where $\mathbf{f}_{j}$ is the constant approximation to $\mathbf{f}$ for $\mathbf{x} \in \Gamma_{j}$. Equation (6) yields a $(2 N) \times$ $(2 N)$ linear system of equations that can be written in block matrix form as

$$
A \mathbf{f}=\mathbf{b}
$$

where each $2 \times 2$ block of $A$ and $2 \times 1$ block of the right-hand side vector $\mathbf{b}$ are given by

$$
\begin{gathered}
A_{i j}=\int_{\Gamma_{j}} G\left(\mathbf{x}, \mathbf{x}_{i}\right) d \Gamma(\mathbf{x}) \\
\mathbf{b}_{i}=-\frac{1}{2} \mathbf{u}\left(\mathbf{x}_{i}\right)+\sum_{j=1}^{N} \int_{\Gamma_{j}} T\left(\mathbf{x}, \mathbf{x}_{i}\right) \mathbf{u}(\mathbf{x}) d \Gamma(\mathbf{x})
\end{gathered}
$$

respectively. The entries in the coefficient matrix $A$ and the right-hand side vector $\mathbf{b}$ are all defined in terms of line integrals along part (or all) of the boundary $\Gamma$. When $i \neq j$ in (7) the integrals are non-singular and can be evaluated using a suitable quadrature rule. Here a 10 point Gauss-Legendre rule has been used as the results presented in Section 3 show that this is sufficiently accurate. If $i=j$ in (7) then the integrals are weakly singular and need to be evaluated using special methods. Here each integral is split into two at the weakly singular point and rule based on the change of variables $x=\tanh (u)$ with 20 points is used to evaluate the integral over the two line segments either side of the singular point. Further details on this quadrature rule can be found in Davis and Rabinowitz [19].

It is noted here that the boundary integral equations (5) can be used to compute the surface forces at any given point in time. However, if the fluid velocities are subsequently required at a different time then the full boundary element calculations will have to be carried out for the new time.

Once the surface forces have been calculated, the total drag force acting on the $i^{\text {th }}$ cluster is given by

$$
\int_{\Gamma^{[i]}} \mathbf{f}(\mathbf{x}) d \Gamma(\mathbf{x}) .
$$

In addition to the hydrodynamic drag force acting on the cluster, there will also be a force due each cluster of cells reacting to the concentrations of a chemical in the surrounding fluid. If $C$ denotes the concentration of the chemical in the fluid, then the total force acting on the $i^{\text {th }}$ cluster will be

$$
\mathbf{F}_{i}=\int_{\Gamma^{[i]}}\left(\mathbf{f}(\mathbf{x})+k_{i} \nabla C(\mathbf{x})\right) d \Gamma(\mathbf{x})
$$

where $k_{i}$ is a parameter which controls how strongly the $i^{\text {th }}$ cluster reacts to the chemical gradient. How $C$ (and hence $\nabla C$ ) are calculated depends on whether the chemical simply present in the surrounding fluid or if it is secreted by the cells in the cluster. In the work presented here, the chemical is assumed to simply be present in the surrounding fluid and moves through the fluid as a simple wave and that the motion of the cells and fluid does not affect how the chemical spreads through the fluid. This allows the concentration $C$ to be given by simple formula and the gradient of the concentration can be obtained by simply differentiating this formula. A more sophisticated model 
would make use of the diffusion-convection equation to simulate how the chemical spreads through the moving fluid medium. However, this would require the governing differential equation to be solved at each time-step using a numerical method such as the finite element method, and the computational cost of doing this can be prohibitive.

Once the total force acting on the clusters has been calculated, the acceleration of each cluster can be found using

$$
\frac{d \mathbf{v}_{i}}{d t}=\frac{1}{m_{i}} \mathbf{F}_{i}
$$

where $m_{i}$ denotes the mass of the cluster.

The torque acting at a point on boundary of the $i^{\text {th }}$ cluster is given by

$$
\tau(\mathbf{x})=\left(x-x_{i}\right)\left(\mathbf{f}_{y}(\mathbf{x})+k_{i} \frac{\partial C}{\partial y}\right)+\left(y-y_{i}\right)\left(\mathbf{f}_{x}(\mathbf{x})+k_{i} \frac{\partial C}{\partial x}\right) \quad x \in \Gamma^{[i]}
$$

and hence the angular acceleration of the cluster is

$$
\alpha_{i}=\frac{1}{I_{i}}\left(\int_{\Gamma^{[i]}} \tau(\mathbf{x}) d \Gamma(\mathbf{x})\right)
$$

where $I_{i}$ denotes the moment of inertia of the cluster.

The motion of each cluster of cells can be characterised by the motion of the centre of mass of each cluster. The location $\mathbf{x}_{i}$ and rotation $\theta_{i}$ of each cluster satisfy the system of ordinary differential equations:

$$
\begin{aligned}
& \text { Cluster location: } & \frac{d \mathbf{x}_{i}}{d t} & =\mathbf{v}_{i} \\
& \text { Cluster Velocity: } & \frac{d \mathbf{v}_{i}}{d t} & =\frac{1}{m_{i}} \mathbf{F}_{i} \\
& \text { Cluster rotation: } & \frac{d \theta_{i}}{d t} & =\omega_{i} \\
& \text { Cluster angular velocity: } & \frac{d \omega_{i}}{d t} & =\alpha_{i}
\end{aligned}
$$

where the force on the cluster is given by (8) and the angular acceleration is given by (9). The coupled system of ordinary differential equations (10), subject to suitable initial condition, can be integrated through time using a suitable numerical method. Here a fourth-order Runge-Kutta method has been used as it is well known that the method has excellent stability and accuracy properties (see one of the many texts on numerical methods, such as Atkinson [20], for further details of the Runge-Kutta method).

\section{Numerical Results}

For all of the numerical results presented here the outer boundary is a square with sides of length 5000 units which corresponds to a square petri-dish with sides of length $5 \mathrm{~cm}$. 
The first example considered is the motion of circular cluster, of radius one unit, in response to the chemical wave moving parallel to the $y$ axis and given by

$$
C(x, y, t)=\frac{1}{4}[1+\tanh (2(t / 4-y-5))] .
$$

The concentration of the chemical in front of the wave is zero, and after the wave has passed the chemical concentration is 0.5 , corresponding to the fluid being saturated. The gradient of the chemical signal can be found by simply differentiating (11) to give

$$
\nabla C=\left[\begin{array}{l}
\frac{\partial C}{\partial x} \\
\frac{\partial C}{\partial y}
\end{array}\right]=\left[\begin{array}{c}
0 \\
\frac{\tanh ^{2}(t / 2-2 y-10)-1}{2}
\end{array}\right] .
$$

This problem has been solved using different numbers of boundary elements, and the details of the meshes used are summarised in Table 1. In each case a time-step of 0.05 units of time was used as the effect of reducing the length of the time-steps on the results was much less than the effect of increasing the number of boundary elements. The numerical results show that the cluster only moves in the $y$ direction, as expected,

\begin{tabular}{|c|c|c|}
\hline $\begin{array}{c}\text { Mesh } \\
\text { Reference }\end{array}$ & $\begin{array}{c}\text { Number Of Elements On } \\
\text { The Cluster Boundary }\end{array}$ & $\begin{array}{c}\text { Number Of Elements On } \\
\text { The Outer Boundary }\end{array}$ \\
\hline A & 40 & 160 \\
B & 80 & 160 \\
C & 160 & 160 \\
FINE & 500 & 800 \\
\hline
\end{tabular}

Table 1: The number of boundary elements on the boundary of the circular cluster and outer boundary for each mesh used.

as that is the direction in which chemical signal is moving.

Figure 1 shows the $y$ coordinate of the centre of the circular cluster when each of the meshes $\mathrm{A}, \mathrm{B}$ and $\mathrm{C}$ have been used to solve the governing integral equation (6). The curves are superimposed on each other, indicating that the numerical solutions obtained using each mesh are almost identical. As can be seen, the cluster gets an initial acceleration as the front of the chemical wave passes, but then the viscous forces in the fluid cause the cluster to slow down and stop again. As expected, there is no motion in the $x$-direction. Within the simulation, the typical distance that a cluster moved in the $x$-direction was of the order of $10^{-12}$ units, which can be considered to be zero to the precision of the computer used to carry out the calculations.

Figure 2 shows the difference between the calculated $y$ coordinate of the centre of the cluster computed using each of meshes A, B and C and the $y$ coordinate calculated using the FINE mesh. As the number of boundary elements increases, the difference between the locations calculated using meshes $\mathrm{A}, \mathrm{B}$ and $\mathrm{C}$ and the solution obtained using the FINE mesh is decreasing and the number of elements increases, and that these differences are much smaller that the size of the vertical displacements as shown 
in Figure 1. These graphs show that the boundary integral method is converging for this example and yielding accurate results.

The second example is considers the motion of two irregular shaped clusters moving under the influence of a circular chemical wave emitted from a point above and to the left of the location of the cells. The larger of the two clusters (see Figure 3) is modelled as being at the origin of the non-dimensional coordinate system used and the smaller cluster is initially located at the point with non-dimensional coordinates $(5.7,-9.7)$. Here the density of the cells is 6 times that of the fluid and the viscosity parameter is $5 \times 10^{-4}$.

In this case the concentrations are given by

$$
C(x, y, t)=\frac{A}{4}\left[1+\tanh \left(2\left[\sqrt{\left.\left(x-x_{0}\right)^{2}+\left(y-y_{0}\right)^{2}\right)}-20 t / 3\right]\right)\right]
$$

where $\left(x_{0}, y_{0}\right)$ are the coordinates of the point from which the circular wave is being emitted. In the results presented here the centre of the wave is located at the point with non-dimensional coordinates $(-5,10)$, the amplitude $A$ was set to 0.12 and for both clusters the reaction rate $k_{i}$ was set to 1 .

Figure 3 shows the results of using the boundary integral model to simulate the motion of some clusters of cells that have been filmed during an experiment (the video frames are courtesy of the Brighton Centre for Regenerative Medicine). Figure 3(a) shows the two simulated clusters in their initial positions with the red lines representing the boundaries of the clusters used by the boundary integral method to model the motion. Figure 3(b) shows the positions of the clusters after $60 \%$ of the time between the first and final positions, and Figure 3(c) shows the final positions of the clusters. The other small clusters and single cells that can be seen in these figures were not included in the boundary integral model. Here two different boundary element meshes were used for each cluster and a smaller time-step was used with the finer boundary element mesh. In Figure 3 the results for the coarse mesh are plotted in blue and the fine mesh in red, but as the locations of the two meshes are superimposed only the results for the fine mesh are visible. This indicates that the boundary integral method is again yielding accurate results in the sense that the numerical approximation errors in the boundary element method are small. When comparing the simulation to the experimentally observed clusters, these results show that the boundary integral model has been reasonably successful at simulating the translational motion of both of the clusters, although the match as the intermediate step is not as good as match at the final step. Also, the model has not been so good at simulating the rotation of the larger cluster. It is possible that these discrepancies may be due to the assumption that the clusters have uniform density not being valid. This means that the location of the centre of mass and moment of inertia of each cluster may not have been calculated very accurately.

\section{Improvements and Future Work}

A key improvement to the current model is to develop a method for accurately determining the centre of mass and moment of inertia of a cluster of cells. A method 


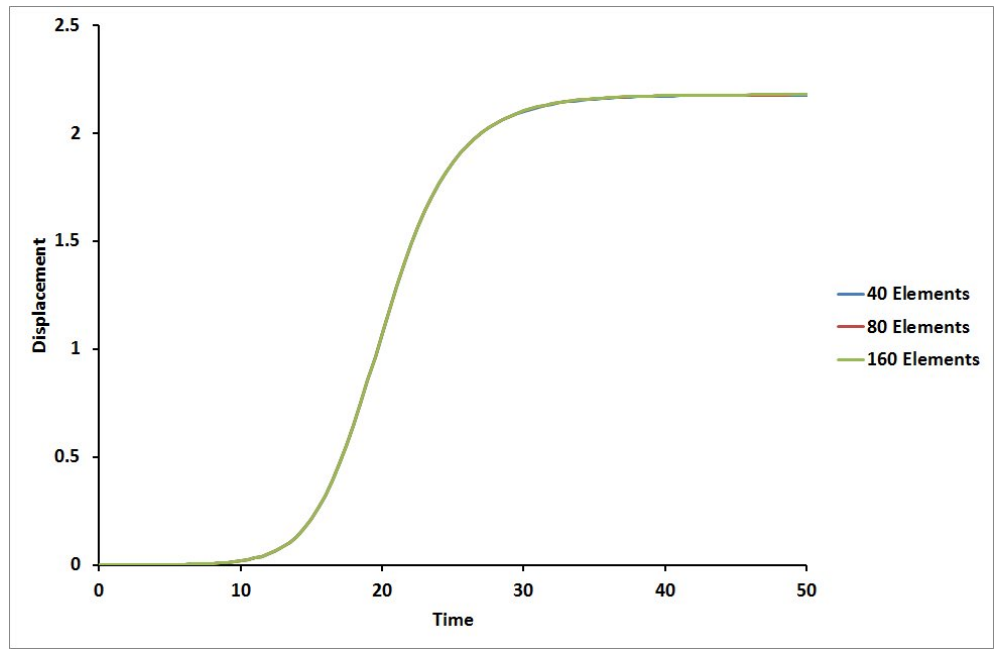

Figure 1: The vertical displacement of the centre of a circular cluster using different numbers of boundary elements.

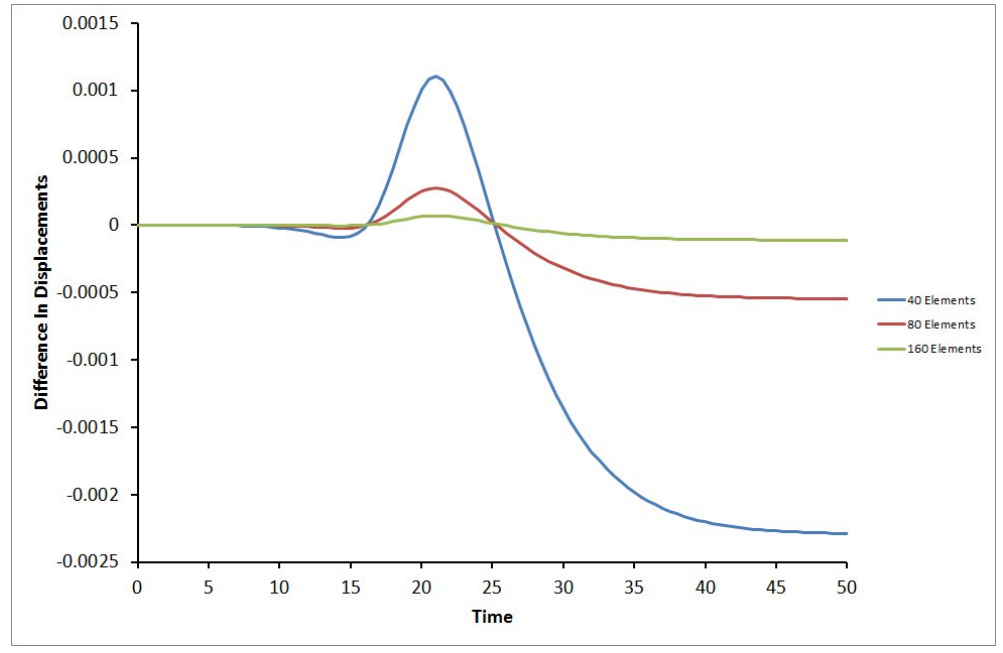

Figure 2: The difference between the displacement of the centre of a circular cluster for different number of boundary element when compared to the displacements calculated using the mesh with 500 elements.

based on image segmentation could be used to identify the location and shape of individual cells contained within a cluster which can be used to compute a better estimate of the clusters the centre of mass and moment of inertia. However, it is possible that the mass of the material contained within each cell is not uniformly distributed within a cell and much more sophisticated model is needed to determine the centre of mass and moment of inertia in such cases.

The results presented in the paper show that a Stokes flow model can be used to 


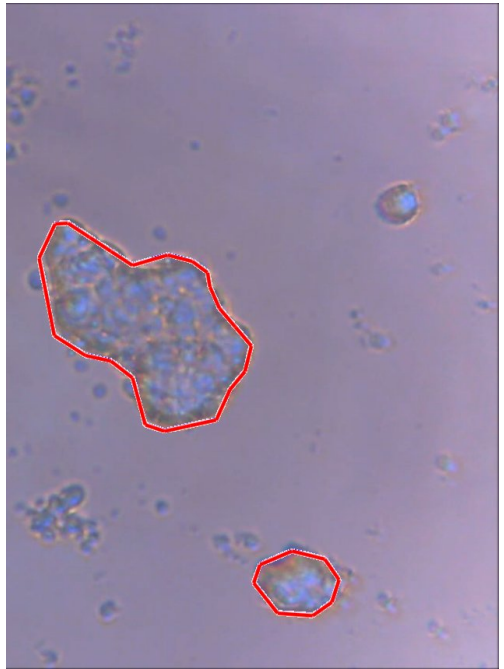

(a) Initial Cluster Locations

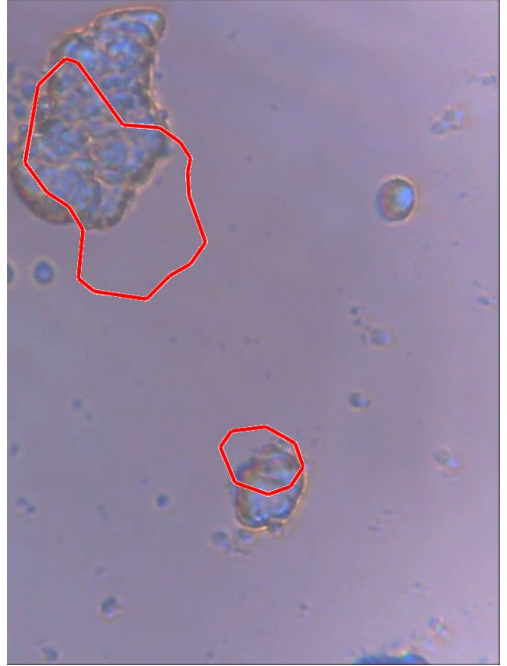

(b) Intermediate Cluster Locations

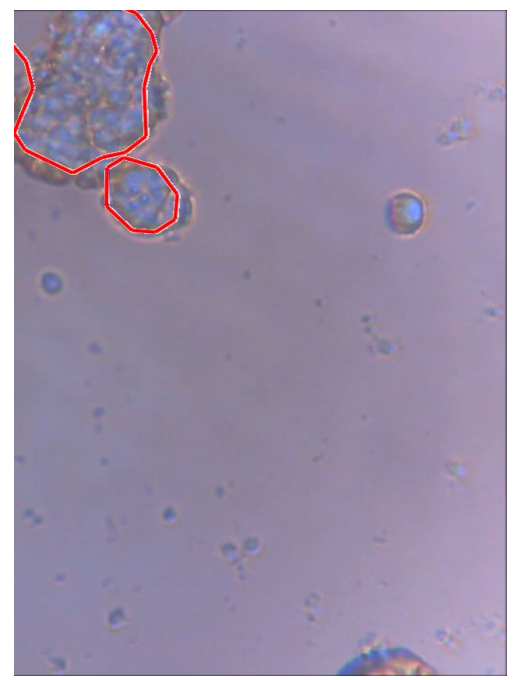

(c) Final Cluster Locations

Figure 3: The results of applying the boundary integral model to simulate the motion of real clusters of cells. The video frame are courtesy of the Brighton Centre for Regenerative Medicine.

simulate the motion of clusters of cells moving through a viscous fluid due to chemotaxis which driven by a chemical spreading through the fluid. However, the spread of the chemical that signals the cells through the fluid will be affected by the motion of the fluid and this can be modelled by the convection-diffusion equation. Ideally, the boundary integral method would also be used to solve this equation. However, whilst boundary integral equations for solving the diffusion equations on a fixed domain have 
been known for many years, (see [21, 22] for example), as far as the author is aware there are no boundary integral methods for solving the convection-diffusion equation with an arbitrary fluid flow field or where the shape of the domain of the underlying differential equation is changing. The alternative is to use a finite element based method to solve the diffusion-convection equation but there are a couple of potential problems with using this method that need to be overcome. Firstly, as the clusters are moving relative to one another the fluid domain would need to be re-meshed at each time-step. Secondly, the solution would need to be interpolated from the old mesh to the new mesh and this could be computationally expensive to implement. Alternatively, it may be possible to model the cells as moving over a fixed finite element mesh and by allowing the chemical to diffuse through the cells as well as the surrounding fluid it would be possible to avoid having to remesh the fluid domain and eliminate the interpolation problems.

The present model can only continue a calculation up to the point where two (or more) clusters collide, and model of how the cells adhere to each other needs to be developed. A starting point may be to consider how fluid droplets combine and coalesce as many mathematical models of the way in which two drops of fluid coalesce to form a single, larger drop have been developed (see [23, 24, 25] for example). A drawback of using these methods is that as the fluid droplets coalesce the masses of fluid within each droplet combine and mix to form the single mass of fluid within the final droplet, but biological cells do not mix in this way as the material contained within each cell remains separate within the membranes of each cell. However, the early stages of two fluid droplets colliding before the fluid in the droplets has started to mix might form a starting point for modelling how two cluster of cells collide and adhere to each other to form a larger cluster.

\section{Conclusions}

This paper has presented a Stoke-flow model of how clusters of cells move through a fluid and used the boundary integral method to solve the resulting differential equations in space and the Runge-Kutta method to integrate the system through time. The results for the single circular cluster demonstrates that the numerical methods described here are yielding accurate solutions to the underlying differential equations. The model was then used to simulate the motion of two cell clusters as observed in an experiment, and the results presented in this paper show that model was able to simulate the translational motion of the two clusters. However, the rotation of one of the clusters was not simulated so well, but this may be due to the assumption that the cluster has a uniform density which may not be the case.

\section{Acknowledgment}

The author would like to thank Matteo Santin and Gary Phillips from The Brighton Centre for Regenerative Medicine for their help and advice with some of the biological aspects of this paper. 
[1] S. Malawista, A. Chevance, L. Boxer, Random locomotion and chemotaxis of human blood polymorphonuclear leukocytes from a patient with Leukocyte Adhesion Deficiency-1: Normal displacement in close quarters via chimneying, Cell Motility and the Cytoskeleton 46 (3) (2000) 183-189.

[2] O. Hoeller, R. Kay, Chemotaxis in the absence of pip3 gradients, Current Biology 17 (9) (2007) $813-817$.

[3] N. Nitta, T. Tsuchiya, A. Yamauchi, T. Tamatani, S. Kanegasaki, Quantitative analysis of eosinophil chemotaxis tracked using a novel optical device taxiscan, Journal of Immunological Methods 320 (12) (2007) 155 - 163.

[4] L. Laganenka, R. Colin, V. Sourjik, Chemotaxis towards autoinducer 2 mediates autoaggregation in Escherichia coli, NATURE COMMUNICATIONS 7. doi:10.1038/ncomms12984.

[5] D. Jia, D. Dajusta, R. Foty, Tissue surface tensions guide in vitro self-assembly of rodent pancreatic islet cells, Developmental Dynamics 236 (8) (2007) 20392049. doi:10.1002/dvdy.21207.

[6] J. Hilderink, S. Spijker, F. Carlotti, L. Lange, M. Engelse, C. van Blitterswijk, E. de Koning, M. Karperien, A. van Apeldoorn, Controlled aggregation of primary human pancreatic islet cells leads to glucose-responsive pseudoislets comparable to native islets, Journal Of Cellular And Molecular Medicine 19 (8) (2015) 1836-1846. doi:10.1111/jcmm.12555.

[7] E. Keller, L. Segel, Model for chemotaxis, J. Theor. Biol. 30 (1971) 225 - 234.

[8] I. Lapidus, R. Schiller, A mathematical model for bacterial chemotaxis, Biophys. J. 14 (1974) $825-834$.

[9] H. Gajewski, K. Zacharias, Global behaviour of a reaction - diffusion system modelling chemotaxis, Math. Nachr 195 (1998) 77 - 114.

[10] A. Chertock, A. Kurganov, X. Wang, Y. Wu, On a chemotaxis model with saturated chemotactic flux, Kin. and Rel. Mod 5 (1) (2012) 51 - 95.

[11] Y. Deleuze, C. Chiang, M. Thiriet, T. Sheu, Numerical study of plume patterns in a chemotaxis-diffusion-convection coupling system, Computers \& Fluids 126 (2016) 58-70. doi:10.1016/j.compfluid.2015.10.018.

[12] M. Eyiyurekli, P. Manley, P. Lelkes, D. Breen, A computational model of chemotaxis-based cell aggregation, BioSysytems 93 (2008) 226 - 239.

[13] R. Thompson, C. Yates, R. Baker, Modelling cell migration and adhesion during development, Bull. Math. Biol. 74 (2012) 2793 - 2809.

[14] M. Kim, D. Reed, K. Rejniak, The formation of tight tumor clusters affects the efficacy of cell cycle inhibitors: A hybrid model study, J. Theor. Biol. 352 (2014) $31-50$. 
[15] P. J. Harris, A simple mathematical model of cell clustering by chemotaxis, Mathematical Biosciences 294 (2017) 62 - 70 . doi:https://doi.org/10.1016/j.mbs.2017.10.008.

URL https://www.sciencedirect.com/science/article/pii/S0025556417305497

[16] C. Elliott, B. Stinner, C. Venkataraman, Modelling cell motility and chemotaxis with evolving surface finite elements, J. R. Soc. Interface 9 (2012) 3027 - 3044.

[17] M. Lighthill, An informal introduction to theoretical fluid mechanics, Clarendon Press, Oxford, 1986.

[18] C. Pozrikidis, Boundary Integral and Singularity Methods for Linearized Viscous Flow, Cambridge Texts in Applied Mathematics, Cambridge University Press, 1992.

URL https://books.google.co.uk/books?id=qXt5bOqDEgQC

[19] P. J. Davis, P. Rabinowitz, Methods of numerical integration, 2nd Edition, Academic Press, London, 1984.

[20] K. Atkinson, An introduction to numerical analysis, 2nd Edition, John Wiley and Sons, New York, 1989.

[21] DAS Curran, BA Lewis and M Cross, A boundary element method for the solution of the transient diffusion equation in 2 dimensions, Applied Mathematical Modelling, 10, 1986, $107-113$.

[22] EA McIntyre, Boundary integral solutions of the heat-equation, Mathematics Of Computation, 46, (1986), 71 - 79.

[23] N Nikolopoulos, A Theodorakakos and G Bergeles. Off-centre binary collision of droplets: A numerical investigation, International Journal of Heat and Mass Transfer, 52, (2009), $4160-4174$.

[24] M Mohammadi, S Shahhosseini and M Bayat. Direct numerical simulation of water droplet coalescence in the oil. International Journal of Heat and Fluid Flow, $36,(2012) 58-71$.

[25] Y R Zhang, X Z Jiang and K H Luo. Bounce regime of droplet collisions: A molecular dynamics study. Journal of Computational Science, 17, (2016), 457 462. 